\title{
Towards Efficient, Broad and Accessible Electronic Scientific Publication
}

\author{
Ayman Karkar \\ Department of Nephrology, Kanoo Kidney Center, Dammam Medical Complex, PO Box 11825, Dammam 31463, Saudi Arabia
}

The rapid and continuous innovations and improvements in basic and clinical research have led to huge treasure of scientific achievements, which are passionately awaited by scientists, physicians and surgeons to fill full the needs of progressing research and medical practice applications. This is particularly the case in kidney transplantation, where, since the end of last century, there has been tremendous basic and clinical research which led to great improvements in immunosuppressive management, surgical techniques, and perioperative care of the transplant candidate and recipient. The ability to cope with such large sum of information continues to require an increasing demand for scientific journals and necessitates the need to speed up the process of publication, distribution and easy and free accessibility to these changes. However, conventional medical journals have limited capacity in terms of reviewing process, space allowed for published work, number of published articles, increasing costs, subscriptions fees to access journals, and time required for publication and dissemination of the articles. Recently, many basic scientific and medical/surgical journals have been switching partially or completely into online service trying to cope with such challenges, yet they are still limited to the scope of field of publication and requirement of subscription fees in order to access such journals.

OMICS Publishing Group is an Open Access Publisher and scientific Events Organizer for the Advancement of Science and Technology. In fact, OMICS Publishing Group is an Open Access publication model that strongly supports the Bethesda Statement on Open Access Publishing and enables the dissemination of research articles to the global community, where it can be accessed by anyone. Abstracts and full texts (HTML, XML and PDF format) of all articles published work are freely accessible to everyone immediately after publication. All works published by OMICS Publishing Group are under the terms of the Creative Commons Attribution License. This permits anyone to copy, distribute, transmit and adapt the work, provided that the original work and source is appropriately cited. Papers published in OMICS Publishing Group are peer reviewed taking in consideration, as well, keeping up to date with latest advances in the respective fields and papers published will reach the readers directly through email. The archived online transactions of OMICS Publishing Group provide the International scientific community with immediate and permanent access to individual papers. OMICS Publishing Group accepts online letters from the Editors and brief comments that contribute to the previously published articles or other relevant findings in OMICS field. OMICS Publishing Group services include Special Features -OMICS Group Journals, Digital Articles, Audio Version-Enhanced Feature of its Publications, Language Translation, Social networking, and Special Features -OMICS Group Conferences (http://www.omicsonline. org/special-features.php). Among the wide scope of OMICS Group Journals, is the newly released specialized journal in organ transplantation "Transplantation Technologies \& Research Journal".

Transplantation Technologies \& Research Journal is a broad-based journal which was founded on key tenets including publishing the most exciting researches with respect to the subjects of Transplantation Technologies, providing a rapid turn-around time possible for reviewing and publishing, and disseminating the articles freely for research, teaching and reference purposes. It was designed to provide transplantation surgeon, physician and scientist a complete source of authentic information about the current developments in the field of Transplantation Technologies, advance our understanding of transplantation research, showing the advancements in the field of regenerative medicine, and publishes research results covering a very broad range of organ transplantation and transplant immunology. Transplantation Technologies \& Research Journal is under open access category using online manuscript submission. This allows the Journal to be promptly available and freely accessible globally through internet to share the innovations of the researchers for scholarly advancement in this field. The review process and tracking systems by the Editorial Manager and the editorial board members, or outside experts (at least two independent reviewers approval followed by editor approval is required for acceptance of any citable manuscript) will ensure quality and quick review processing. Transplantation Technologies $\&$ Research Journal allows the transplant specialist to keep up to date on the latest developments in the research areas pertaining to specific organ systems, mechanisms of rejection, tolerance induction and xenotransplantation. Transplantation Technologies \& Research Journal has been most fortunate to have a wide number of qualified editors who will have invaluable contributions to this journal and who will attempt to continue to provide you with the latest developments in the field of transplantation. Here, the Editorial Board are pleased to welcome you and introduce you to the first issue of this international peer-reviewed open access journal the "Transplantation Technologies \& Research Journal".
Corresponding author: Ayman Karkar, PhD, FRCP, Department of Nephrology, Kanoo Kidney Center, Dammam Medical Complex, PO Box 11825, Dammam 31463, Saudi Arabia, Email: aymankarkar@yahoo.com

Received July 09, 2011; Accepted July 14, 2011; Published September 26, 2011

Citation: Karkar A (2011) Towards Efficient, Broad and Accessible Electronic Scientific Publication. J Transplant Technol Res 1:104e. doi:10.4172/21610991.1000104e

Copyright: ( ) Karkar A. This is an open-access article distributed under the terms of the Creative Commons Attribution License, which permits unrestricted use, distribution, and reproduction in any medium, provided the original author and source are credited. 